\title{
Investigation of the Neutral Gas Pressure Effect on the Metal Resistive Bolometer
}

\author{
D. Zhang, L. Giannone, O. Grulke*, M. Piechotka, T. Windisch, A. Stark*, \\ T. Klinger
}

\author{
Max-Planck-Institut für Plasmaphysik, EURATOM Association, Wendelsteinstr. 1, \\ 17491 Greifswald, Germany \\ *Ernst-Moritz-Arndt Universität, 17491 Greifswald, Germany
}

\begin{abstract}
The bolometer system planned for W7-X consists mainly of metal (Au) resistive detector arrays. All the detectors are exposed to neutral gas environment. The thin bolometer foil used for detecting the radiated power loss may be sensitive to the neutral gas pressure due to the strain gauge effect. Recently, a prototype of this kind of bolometer camera consisting of 12 channels has been installed on the cylindrical plasma device VINETA in order to investigate the influences of the neutral gas pressure on the bolometer signals. Experiments are carried out for Ar-discharges under different gas pressure conditions. It is found that the pressure effect of the neutral gas can make considerable contributions, thus inducing non-negligible errors of the results in most of the investigated cases. Using the VINETA plasmas ( $\mathrm{Ar}, \mathrm{T}_{\mathrm{e}}<10 \mathrm{eV}, \mathrm{n}_{\mathrm{e}}<10^{-19} \mathrm{~m}^{-3}$ ) as examples, the paper demonstrates and discusses how to minimize the neutral gas effects, especially in the data analysis process. The radiated power and the radiation intensity profile obtained in helicon discharges are presented.
\end{abstract}

Keywords: metal resistive bolometer, neutral gas pressure effect, strain gauge effect, bias, measurement error, radiated power loss, spatial radiation distribution, W7-X, VINETA

PACS: 52.70.-m, 52.25.Os, 52.50.Qt, 52.80.Pi

\section{INTRODUCTION}

The bolometer system planned for W7-X consists mainly of metal (Au) resistive detector arrays [1] already widely used for other devices [2-4]. In addition to measuring the total radiated power, the bolometer system is capable of performing tomographic reconstruction of spatial radiation distribution. The reconstructed profiles from inversion processes depend strongly on the accuracy of the raw, line-integrated signals. Hence discriminating and minimizing the 'error' sources are essential for improving the bolometric results.

The raw signal of the bolometer results from an imbalance among the four branches of an AC-powered Wheatstone bridge. Two resistors $\left(R_{m}\right)$ as sensors (absorber) are exposed to plasmas while the other two $\left(R_{r}\right)$ as reference are shielded by a front plate. The resistance of the detector is sensitive to the power load from plasma radiation, being the principle of the resistive bolometer. However, working environment can affect the bolometer measurement, e.g. stray radiation from ECR-heating system and neutral gas. Neutral gas pressure causes so-called strain-gauge effect [5] due to the sensitivity of the meander resistance to the inner strain. This effect has been investigated in the environment provided by the cylindrical linearly magnetized device VINETA [6]. The paper presents the experimental results. Analyzed and discussed are the strain-gauge factor, the level of the bias related to the VINETA-plasma radiation as well as the methods to subtract this contribution from the line-integral. The experience gained is transferred to the current design as well as to the future data analysis process of the W7-X bolometer.

\section{EXPERIMENTAL SET-UP}


A prototype of the bolometer cameras designed for W7-X was used for this study. The camera consists of 12 channels of gold resistive detectors, having $4 \mu \mathrm{m}$-gold-foil absorber coated on $7.5 \mu \mathrm{m}$-Kapton substrate. The detectors are integrated into three bolometer heads. Every four channels share a common front plate to shield the reference foil-resistors. The front plate for channels 5-8 is specifically constructed with four inclined boreholes to compensate the neutral gas pressure effect. On the detector holder, a Pt100 thermometer is mounted to monitor the temperature change.

The camera was installed into a rectangular port on VINETA [figure 1 (a)]. The vacuum chamber has a diameter of $40 \mathrm{~cm}$ and a total length of $4.5 \mathrm{~m}$. The lines of sight of the camera are arranged up/down symmetrically with respect to the midplane and cover the whole plasma cross-section (minor radias $\sim 7.5 \mathrm{~cm}$ ) [figure 1 (b)]. The spatial resolution at the plasma centre is about $2 \mathrm{~cm}$.

In experiments, Ar-plasma is generated by rf-heating $\left(f_{\mathrm{rf}}=13.56 \mathrm{MHz}, \mathrm{P}_{\mathrm{rf}}<4.5 \mathrm{~kW}\right)$. The magnetic field on the axis is set to be $100 \mathrm{mT}$. The required neutral gas pressure is realized through controlling the gas-flow and utilizing a turbo-molecular and rotary pumping system, simultaneously. A capacitive manometer (MKS Baratron ${ }^{\circledR}$ type 627B) is used to measure it. For helicon discharge related, electron density profile is peaked, having a maximum $n_{e}$ on the machine axis. The plasma temperature $\mathrm{T}_{\mathrm{e}}$ is less than $10 \mathrm{eV}[6,8]$.

a

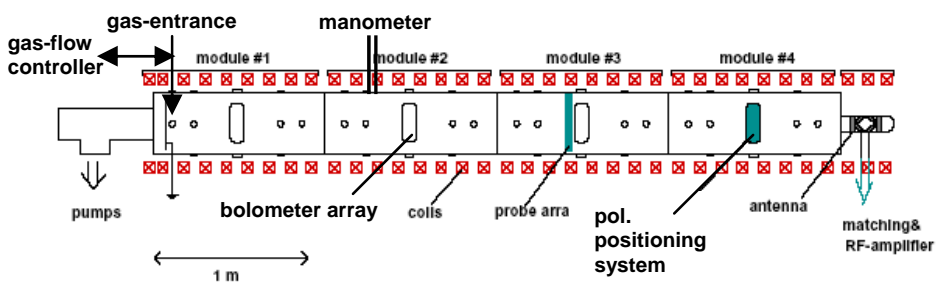

b

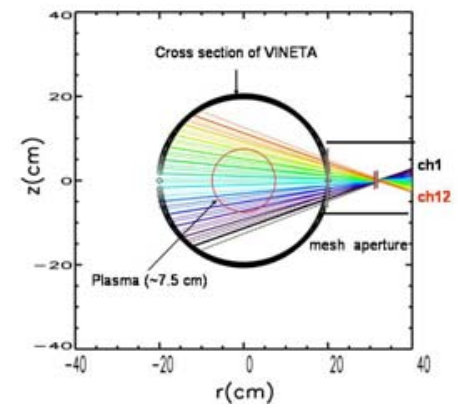

FIGURE 1. a) Schematics of the VINETA device.

b) The line of sight of the 12-channel bolometer camera.

\section{EXPERIMENTAL RESULTS}

\section{Neutral Gas Pressure Effect}

Performing neutral gas pressure scan with certain scan rates $\mathrm{dP}_{\mathrm{n}} / \mathrm{dt}$, bolometer signals, associated with straingauge effect, are measured. Decrease of $\mathrm{P}_{\mathrm{n}}$ leads to increment of the bolometer signal $\mathrm{U}_{\mathrm{d}}$, being the effect on all of the investigated channels. Response functions, $\mathrm{dU}_{\mathrm{d}} / \mathrm{dP}_{\mathrm{n}}<0$, of different values, are obtained, depending on the character of the substrate $(7.5 \mu \mathrm{m}$-Kapton) as well as the inner structure of the gold meander resistor concerned. Since $U_{d}$ is proportional to the relative change of the meander resistance $\Delta\left(R_{m}-R_{r}\right) / R_{0}$, where $R_{0}=\left(R_{m}+R_{r}\right) / 2$, decrease of $P_{n}$ induces increment of $\Delta\left(R_{m}-R_{r}\right)$. Taking two channels (ch2\&8) as examples, figure 2(a) show the experimental data as functions of the Ar-gas pressure $P_{n}$, with $U_{d}$ on the right axis and the corresponding $\Delta\left(R_{m}{ }^{-}\right.$ $\left.\mathrm{R}_{\mathrm{r}}\right) / \mathrm{R}_{0}$ on the left one, respectively. The data of each channel are approximately fitted with a linear function. The slope of the fit reflects the magnitude of the neutral gas effect. Strain-gauge factors, defined as

$$
k_{s g}=\frac{1}{R_{0}} \frac{\Delta\left(R_{m}-R_{r}\right)}{\Delta P_{n}},
$$

are obtained for all detectors. They are in the range of $0.001-0.015 \mathrm{mbar}^{-1}$. The results of ch5-8 indicate that the inclined borehole structure on the front plate can not compensate the neutral gas pressure effect absolutely.

Extending the scan range of $\mathrm{P}_{\mathrm{n}}$ and performing the scan up/down continuously, hysteresis effect is observed, which is demonstrated in figure 2(b). This effect is probably induced through the larger scan rate $\mathrm{dP}_{\mathrm{n}} / \mathrm{dt}$, leading to an irreversible strain change of the meander resistor. 

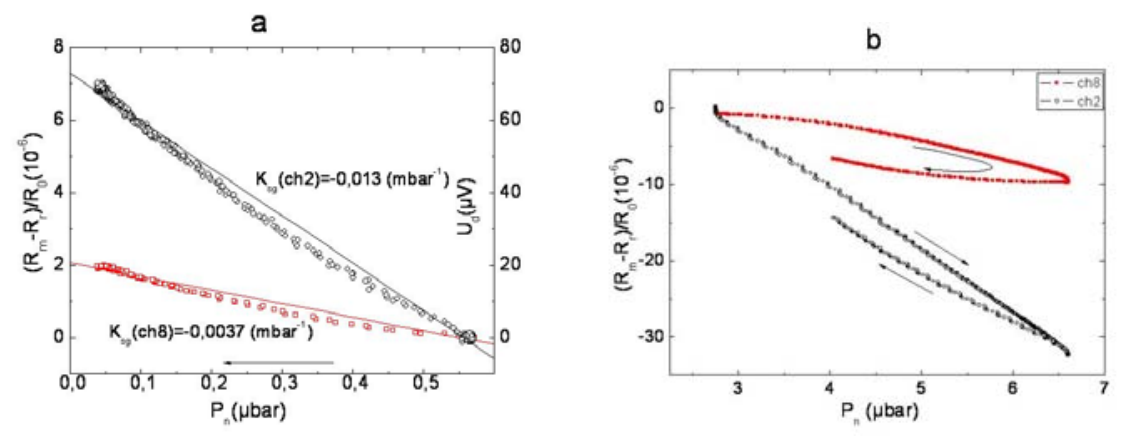

FIGURE 2. a) Bolometer signal $U_{d}$ (right axis) and the corresponded relative resistance change of the gold meander resistors (left axis) arisen from neutral gas pressure effect. b) Hysterisis effect of the neutral gas pressure on the detectors.

\section{Contribution of the Neutral Gas Pressure Effect to the Line-integral}

Performing helicon Ar-discharges at VINETA, the bolometer array is used to measure the radiated power from the plasma. The neutral gas pressure is stabilized before starting rf-heating process. The value of $\mathrm{P}_{\mathrm{n}}$, near to the detectors, is monitored.

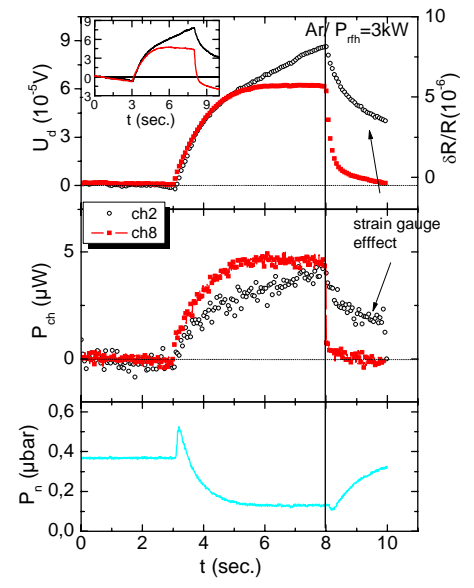

FIGURE 3. The temporal evolution of the bolometer signals $U_{d}$ (upper), the line-integrated power $P_{c h}(\mathrm{mid})$, containing bias from neutral gas pressure effect, and the neutral gas pressure $P_{n}$ for the helicon discharge at VINETA. In the inset are the raw signals $U_{d}$ with slight offset-drift.

Taking ch2 and ch8 again as examples, figure 3 shows the temporal evolution of the data obtained for the discharge with rf-heating power of around $3 \mathrm{~kW}$. The bolometer signals $U_{d}$ and the neutral gas pressure $P_{n}$ are indicated in the upper and the lower part, respectively. The raw data of $U_{d}$, containing slight offset-drift, is presented in the inset, which are corrected through fitting the data before rf-heating with exponential decay functions. The line-integrals of the 'radiated' power $\mathrm{P}_{\mathrm{ch}}$ are shown in the mid picture, which are calculated with the bolometer equation using the detector parameters obtained through the in-situ calibration performed after discharging [3]. The neutral gas pressure $P_{n}$ decreases after the initial phase of the discharge due to the ionization process. It recovers afterwards gradually in a time scale around 2 sec. after turning-off the heating. During this period $\mathrm{P}_{c h}$ shows also time-dependent behaviour, correlated with the variation of $\mathrm{P}_{\mathrm{n}}(\mathrm{t})$. This phenomenon confirms the neutral gas pressure effect again. According to the strain-gauge factors $\mathrm{K}_{\mathrm{sg}}$ obtained (see the section above), the contributions of the neutral gas pressure effect during the discharge are evaluated. The resulted bias for the case of ch2 is comparable to the line-integrated radiation power, roughly around $100 \%$. For the case of ch8 it is around $12 \%$.

It is noteworthy that the influence of the holder temperature to the bolometer signal is excluded, since no temperature change is observed according to the data of the pt100 thermometer. 


\section{The Radiation Profile and the Radiation Level}

Performing the data analysis process described above, the bias arisen from the neutral gas pressure effect are subtracted. The line-integrals from the plasma radiation are obtained. Figure 4(a) shows the results obtained for the discharges with different rf-heating powers. The line-integrated radiation power flux is plotted as a function of the impact radius, i.e. the normal distance from the VINETA-axis to the central sightline. Radiation intensity profiles, obtained through Abel inversion using two different numerical methods, are presented in figure 4 (b) for the discharge with $3.4 \mathrm{~kW}$ heating power. One method is through solving the matrix equations containing the lineintegrals, the geometry function of the channels and the radius-dependent emissivity, directly. The other is a method based on the maximum entropy principle [7]. Maximum radiation intensity around the machine axis is achieved, being consistent with the peaked electron density and temperature profile, measured by electrostatic Langmuir probe [8]. Total radiated power is evaluated through linear extrapolation of the radiated power in the viewed volume of the bolometer array to the whole plasma volume. It is observed, that the total radiated power increases with the rfheating power. Radiation level of around $12 \%$ is achieved.
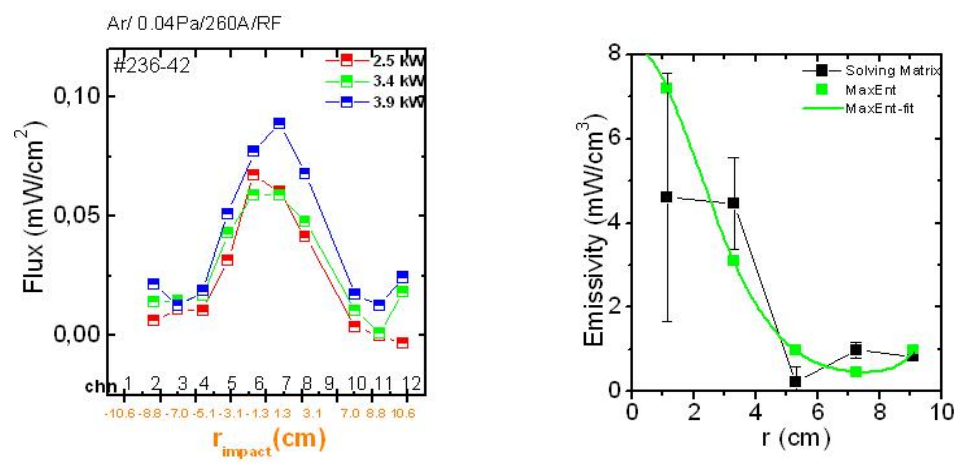

FIGURE 4. The line-integrated radiation flux versus the impact radius for the helicon discharges with three different heating powers (left) and the radiation intensity profiles obtained by Abel inversion (right).

\section{DISCUSSION}

The level of the bias, due to the neutral gas pressure effect, depends on the strain-gauge factor of the detector, the neutral gas pressure change as well as the line-integral from plasma radiation. The experience gained gives an instruction for the current design of the W7-X bolometer system. Two blind channels, lying behind a shield plate against plasma radiation are designed and located near to the detectors. They serve as monitoring the neutral gas pressure effect. The strain-gauge factors of the radiation detectors will be firstly calibrated against the pressure monitors, i.e. the blind channels, according to which the bias will be subtracted.

\section{ACKNOWLEDGMENTS}

We gratefully acknowledge Dr. M. Laux and J. Sachtleben for their invaluable discussion and help concerning this work as well as Ch. von Sehren for designing the bolometer camera.

\section{REFERENCES}

1. D. Zhang, L. Giannone, B. Klein, $34^{\text {th }}$ EPS Conference on Controlled Fusion and Plasma Physics (Warsaw), P1.165, 2007

2. K.F. Mast, J.C. Vallet, C. Andelfinger, P. Betzler, H. Kraus and G. Schramm, Rev. Sci. Instrum. 62, 744 (1991).

3. L. Giannone et al., Review of Scientific Instruments 73, 3205 (2002).

4. K.F. Mast and H. Krause, Review of Scientific Instruments 56, 969 (1985).

5. M.R. Neuman, and W.G. Sutton, J. Vac. Sci. Technol. 6, 710-3 (1969).

6. C.M. Franck, O. Grulke, A. Stark, T. Klinger, E.E. Scime, G. Bonhomme, Plasma Sources Sci. Technol.14, 226 (2005).

7. L. Giannone, A. Elsner, O. Heinrich, M. Hirsch, and the W7-AS team, Review of Scientific Instruments 68, 762 (1997).

8. T. Windisch, “Intermittent events and structure propagation in plasma turbulence”, Ph.D. Thesis, Greifswald University, 2007. 